\title{
Similarities and conformal transformations
}

\author{
by 11. S. Il. Coxersin ('Toronto, Canada)
}

To Enrico Bompiani on his sciontific Jubulce

\begin{abstract}
Summary. - In ardinary Euclidean space, every isometry that leaces no point invariant is either a screw displacement (uncluding a translation as a special case) or a glide reflection. Every other himl of similarity is a spiral similarity: the product of a rotation about a linc and a dilatation rhose center lies on this line. In real inversive space (i.e., Euclidean space plus a single point at infinity). every conformal transtor. mation is either " similarity or the product of an incersion and an isometry. This last remark remains valid when the number of dimensions is increased. In fact. every con. formal tranformution of inversive $n$-space $(n-2)$ is expressible as the proddct of $r$ reflections and $s$ incersions, where $r \leq n+1, s \leq 2, r+s \leq n+2$.
\end{abstract}

1. Introduetion. - Aecording to KLELN's Erlanger Programm of 1872, the eriterion that distinguishes one geometry from another is the group of transformations under which the propusitions remain true. In particular, Euclidean geometry is characterized by the group of similarities, and inversive geometry by the group of circle-preserving (or sphere-preserving) transformations. It is thus natural to study the precise nature of the most general similarity and of the most general sphere-preserving transformation. For simplicity, we shall consider three-dimensional spaces, with a brief remark at the end as to what happens in $n$-dimensional spaces.

2. Isometries. - Let us begin by recalling some familiar definitions and theorems.

An isometry is a transformation (of the whole Euclidean space into itself) which preserves length, so that a line-segment $P Q$ is transformed into a congruent segment $P^{\prime} Q^{\prime}$. A reflection is the special kind of isometry which has a triangle of invariant points, and therefore a whole plane of invariant points: the mirror. An isometry is direct (i. e., sense-preserving) or opposite (i. e., sense-reversing) according as it is the product of an even or odd number of reflections.

Every direct isometry, being the product of 4 reflections, is a screw-dis. placement [see Kelvis and Tart 8. $\$ 102$ or Goxeter 3, p. 38], possibly reducing to the produet of just 2 reflections, which is a rotation or a translation according as the 2 mirrors are intersecting or parallel. In particular, the product of 2 perpendicular reflections is a rotation through $\pi$, that is, a half-turn. Every direct isometry with an invariant point is a rotation.

Every opposite isometry, heing the product of 3 reflections, is either a 
flection: the product of a reflection with a translation along the direction of a line lying in the reflecting plane [Coxeten 3, p. 37].

rotatory-inversion (when the 3 mirrors have a common point) or a glide-re.

Every opposite isometry with an invariant point is a rotatory-inversion, possibly reducing to a single reflection or to a central inversion: the isometry that replaces each point of a sphere by its antipodes. (The name «central inversion", throngh well established in the literature of crystallography, is perhaps unfortunate. We must be careful to distinguish it from «inversion in a sphere $\$ .1$

Every opposite isometry with no invariant point is a glide-reflection.

3. Similarities. - The theory of isometries can be extended to similarities as follows. A similitary is a transformation tof the whole Euclidean space into itself) which transforms each segment into a segment $\mu$ times as long, where $\mu$ is a constant positive number called the ratio of magnification. When $\mu=1$, the similarity is an isometry. Another important special case is a dilatation, which transforms each line into a parallel line [Artin 1, p. 54]. Two given segments on parallel lines, say $A B$ and $A^{\prime} B^{\prime}$, are related by a unique dilatation $A B \rightarrow A^{\prime} B^{\prime}$, which is an isometry if and only if the vector $A^{\prime} B^{\prime}$ is equal to either $A B$ or $B A$. In the former case we have a translation, in the latter a central inversion (about the common midpoint of $A A^{\prime}$ and $B B^{\prime}$ ).

Two given similar tetrahedra $A B C D, A^{\prime} B^{\prime} C^{\prime} D^{\prime}$ are related by a unique similarity $A B C D \rightarrow A^{\prime} B^{\prime} C^{\prime} D^{\prime}$, which is direct or opposite according as the sense of $A^{\prime} B^{\prime} C^{\prime} D^{\prime}$ agrees or disagrees with that of $A B C D$. In fact, any point $P$ is transformed into the unique point $P^{\prime}$ whose barycentric coordinates referred to $A^{\prime} B^{\prime} C^{\prime} D^{\prime}$ are the same as those of $P$ referred to $A B C D$. If follows that two given similar triangles are related by just two similaritics: ont direct and one opposite.

Theoren 3.1 - Every similarity which is not an isometry has just one invariant point.

Proof. - Since any invariant point of a transformation is also an inva. riant point of the inverse transformation, we lose no generality by conside* ring a similarity

$$
A B C D \rightarrow A^{\prime} B^{\prime} C^{\prime} D^{\prime}
$$

in which $A B C D$ is the larger of the two given similar totrahedra. It $A$ and $A$, coincide, we have already found an invariant point. If not, suppose the similarity transform $A^{\prime}$ into $A^{\prime \prime}, A^{\prime \prime}$ into $A^{\prime \prime \prime}$, and so on. Let $\mu$ denote the ratio of magnification, so that $A^{\prime} B^{\prime}=\mu A B$ and $0<\mu<1$. Then

$$
A^{\prime} A^{\prime \prime}=\mu A A^{\prime}, \quad A^{\prime \prime} A^{\prime \prime \prime}=\mu A^{\prime} A^{\prime \prime}, \ldots
$$

Since $\mu<1$, the sequence of points $A, A^{\prime}, A^{\prime \prime}, \ldots$ converges to a point of 
accumulation $\mathrm{O}$ whose distance from $A$ is

$$
\leq A A^{\prime}+A^{\prime} A^{\prime \prime}+A^{\prime \prime} A^{\prime \prime \prime}+\ldots=(1+\mu+\mu+\ldots) A A^{\prime}=(1-\mu)^{-1} A A^{\prime} .
$$

Since the similitarity transforms $A A^{\prime} A^{\prime \prime} \ldots$ into $A^{\prime} A^{\prime \prime} A^{\prime \prime \prime} \ldots$, which is the same sequence, $O$ is invariant. Finally, there cannot be more than one such point, for, if there where two, the segment formed by them wonld be invariant in. stead of heing multiplied by the proper fraction $\mu$.

To locate $O$, ensider the two similarities, one direct and one opposite, which transform the given triangle $A B C$ into a similar (but not congruent) triangle $A^{\prime} B^{\prime} C^{\prime}$. Suppose $A^{\prime} B^{\prime}=\mu A B$, so that $\mu$ is a positive number different from 1 (not necessarily less than 1). Let $A_{1}$ and $A_{2}$ divide $A A^{\prime}$ internally and externally in the ratio $1: \mu$. Let $B_{1}$ and $B_{2,}, C_{1}$ and $C_{2}$, divide $B B^{\prime}, C C^{\prime}$ in the same manner: Consider the three spheres whose diameters are $A_{1} A_{2}, B_{2} B_{2}$, $C_{1} C_{2}$. These are «spheres of Apollonius": $\because g$. , the first is the locus of points whose distances from $A$ and $A^{\prime}$ are in the ratio $1: \mu$. Iny point $O$ for which

$$
O A^{\prime}=\mu O A, \quad O B^{\prime}=\mu O B, \quad O C^{\prime}=\mu O C
$$

must be on all three spheres. We have alrady estahlished the existence of two such points. Hence the three spheres do in fact intersect, enabling us to locate the invariant points of the two similaritics. Finally, the correct one of the two positions for $O$ is determined by a fourth sphere, with diameter $D_{1} D_{2}$.

In particular, every dilatation which is not a translation has just one invariant point. If this point is $O$, the dilatation is conveniently denoted by $O(\mu)$ or $O(-\mu)$ according as it is direct or opposite. For instance, $O(-1)$ is a central inversion, and of course $O(1)$ is simply the identity.

4. The spiral similarity. - A spiral similarity is the product of a rotation about a line $l$ (the «axis») and a dilatation whose «centre» 0 lies on $l$. Suppose it is the product of a rotation through angle $\alpha$ and a dilatation $O(\mu)$ where, for the moment. We allow $\mu$ to take any value except zero. The following values of $\alpha$ and $\mu$ yield special cases which are familiar:

\begin{tabular}{|rr|l|}
\hline$\alpha$ & $\mu$ & \multicolumn{1}{|c|}{ Similarity } \\
\hline 0 & 1 & Ientity \\
$\pi$ & 1 & Half-turn \\
$x$ & 1 & Rotation \\
$\pi$ & -1 & Reflection \\
0 & -1 & Central inversion \\
$x$ & -1 & Rotatory-inversion \\
0 & $\mu$ & Dilatation \\
\hline
\end{tabular}


We observe that this table includes all kinds of isometry, both direct and opposite, except the screw-displacement and glide-reflection (which have no invariant points). Still more surprisingly, with these same two exceptions, every similarity is a spiral similarity. In fact, we have

Theorem 4.1 - Every similarity is either a screw-displacement, a glide-reflection, or a spiral similarity.

Proof. - Since the case of an isometry is well known, it will suffice to consider a similarity that is not an isometry. Suppose again that it multiplies distances by $\mu$, a positive number different from 1 . If 0 is its invariant point, the similarity is evidently expressible as the product of the dilatation $O( \pm \mu)$ and an isometry for which $O$ is again invariant. By choosing the plus or minus sign according as the given similarity is direct or opposite, we can ensure that the residual isometry is direct. Having an invariant point, this isometry must be a rotation. Thus the given similarity, being the product of a dilatation from 0 and a rotation about a line through 0 , is a spiral simila. rity, as desired.

5. Inversive 3-space. - Clearly, every similarity is a "collineation", tran. sforming ranges into ranges and therefore planes into planes. It is also a «sphere-preserving» transformation. transforming spheres into spheres and therefore circles into circles.

If a plane mirror reflects $P$ into $P^{\prime}$, we may describe the image $P^{\prime}$ as the second intersection of three spheres through $P$ orthogonal to the mirror. If we replace the plane by a sphere, the same description vields the image of $P$ by inversion in the sphere; we call $P^{\prime}$ the inverse of $P$. This analogy motivates an extension of the concept "sphere" according to which we re. gard a plane as a special case: "a spher of infinite radius. "Likewise a line is a special case of a circle. We can now say that any thres distinct points lie on a unique circle, and that any sphere inverts into a sphere. In the same spirit we extend Euclidean space to imersive space hy inventing an ideal point at infinity $O^{\prime}$, which is both a common point and the common centre of all planes, regarded as spheres of infinite radius.

We can now assert that, with respect to a given sphere with centre $O$. every point has an inverse. All the planes throngh 0 , being \& spheres » ortho. gonal to the sphere of inversion, meet again in $O^{\prime}$. the inverse of $O$. (When the centre $O$ is $O^{\prime}$ itself. the sphere of inversion is flat, and the inversion reduces to a reflection.) Thus, in inversive space. inversion is a one-fo-one. transformation without exception.

Since every inversion is a sphere-preserving transformation, so also is any product of inversions. For instance, the product of inversions in two concentric spheres is a dilatation. Let $J_{k}$ denote the inversion in a sphere 
with centre $O$ and radius $k$; then, clearly,

$$
J_{1} J_{k}=\mathrm{O}\left(k^{2}\right) \text {. }
$$

It is natural to ask whether every sphere-preserving transformation (of the whole inversive space into itself) is a product of inversions and, if so, how many inversions may be needed. In fact,

TheOREM 5.2. - Every sphere-preserving transformation of inversive space is either a similarity or the product of an inversion and an isometry.

Proof [Cf. Lagrange 9, p. 7]. - We distinguish two cases, according as the point at infinity is is not, invariant. In the former case, not only are spheres transformed into spheres, and circles into circles, but also planes into planes, and lines into lines. With the help of EvoLID III. 21 we deduce that equality of angles is preserved, and consequently the measurement of angles is preserved. so that every tetrahedron is transformed into a similar tetahedron, and the transformation is a similarity.

If, on the other and, the given transformation $T$ takes an ordinary point $O$ into the point at infinity $O^{\prime}$, we consider the product $J_{1} T$, where $J_{1}$ is the inversion in the unit sphere having this particular point $O$ for its centre. This product $J_{1} T$, leaving $O^{\prime}$ invariant, is a similarity. Let $k^{2}$ be its ratio of. magnification. By 5.1, $J_{1} T$ can be expressed as $J_{1} J_{k} S$, where $S$ is an isometry. Thus

$$
T=J_{k} S
$$

the product of an inversion and an isometry.

As a consequence, we have

Theorem 5.3. - Every sphere-preserving transformation of inversive space can be expressed as the product of $r$ reflections and $s$ inversions, where

$$
r \leq 4, \quad s \leq 2, \quad r+s \leq 5
$$

Proof. - Every isometry is expressible as the product of 3 or 4 reflections [Coxeter 3, p. 36]. Since

$$
\mathrm{O}\left(-k^{2}\right)=\mathrm{O}(-1) \cdot \mathrm{O}\left(k^{2}\right),
$$

a spiral similarity is the product of a rotation or rotatory-inversion and a direct dilatation; thus it is the product of 2 or 3 reflections and 2 inversions. By Theorem 5.2. every other sphere-preserving transformation is the product of 3 or 4 rerlections and an inversion. 
If we wish to use the language of pure inversive geometry, in which the point at infinity is not specialized, we simply say that every sphere-preserving transformation can be expressed as the product of 4 or 5 inversions.

6. Conformal transformations. - A well known theorem of DuPIN states that, for three mutually orthogonal systems of surfaces, the lines of curvature on any surface in one of the systems are its intersections with the surfaces of the other two systems. Since any surface may be exhibited as a member of one of three mutually orthogonal systems, it follows that any conformal (i. e.; angle-preserving) transformation takes lines of curvature into lines of curvature. Since a sphere (including a plane as a special case) is characterized by the property that all directions on it are principal directions, we can immediately deduce LIouviLLE' s Theorem: Every conformal transfor. mation is a sphere-preserving transformation. Thus Theorems 5.2 and 5.3 are still true when we replace "sphere-preserving» by «conformal "[FondER 6, pp. 137-138].

7. Euclidean n-space. - Theorems 3.1 and 5.2 evidently remain valid in any number of dimensions. But Theorem 4.1, in its remarkable simplicity, is peculiar to 3 dimensions. When the number of dimensions is even, the central inversion $\mathrm{O}(-1)$ is direct; therefore direct and opposite similarities have to be treated separately

Let $Q$ denote a rotation, $R$ a reflection, $T$ a translation, and let $Q^{q} R^{r} T$ denote a product of several such transformations, all commutative with one another. In this notation, $R T$ is a glide-reflection (in 2 or 3 dimensions), $Q R$ is a rotatory-inversion, $Q T$ is a serew-displacement, and $Q^{2}$ is a double rotation (in 4 dimensions). The general description of isometries in Euclidean $n$-space seems to have been first given by P. H. SCHOUTE [COXETER 3. p. 218]. In $2 q$ dimensions, every direct isometry is $Q^{q}$ or $Q^{q-1} T$, and every opposite isometry is $Q^{q-1} R T$. In $2 q+1$ dimensions, every direct isometry is $Q^{q} T$, and every opposite isometry is $Q^{q} R$ or $Q^{q-1} R T$. (This description includes all possible special cases, provided we allow the angle of a $Q$ or the distance of a $T$ to be zero.) To obtain the corresponding expressions when there is an invariant point (so that the isometry is an «orthogonal transformation $\gg$ ), we merely have to omit $T$.

Let $D$ denote a dilatation which is commutative with $R$ and with every Q. Then our proof of Theorem 4.1 can be extended to yield

THEOREM 7.1 - In $2 q$ dimensions, every derect similarity is $Q^{q-1} T$ or $Q^{q} D$, and every opposite similarity is $Q^{q-1} R T$ or $Q^{q-1} R D$. In $2 q+1$ dimensions, every direct similarity is $Q^{q} T$ or $Q^{q} D$, and every opposite similarity is $Q^{q-1} R T$ or $Q^{p} D$. 
In $2 q$ dimensions, $D$ is always direct. In $2 q+1$ dimensions, if we insist on a direct $D$ (so that we can use 5.1), the opposite similarity $Q^{q} D$ must be replaced by $Q^{q} R D$.

8. Inversive n-space. - Using Theorem 5.2 and replacing each direct $D$ by the product of inversions in two concentric spheres, we obtain the following numbers of reflections and inversions for the various kinds of conformal transformation:

In $2 q$ dimensions (direct), $2 q$ reflections and 2 inversions, or $2 q+1$ reflections and 1 inversion.

In $2 q$ dimensions (opposite), $2 q+1$ reflections, 1 or 2 of which may be replaced by inversions.

In $2 q+1$ dimensions (direct), $2 q+2$ reflections, 1 or 2 of which may be replaced by inversions.

In $2 q+1$ dimensions (opposite), $2 q+1$ reflections and 2 inversions, or $2 q+2$ reflections and 1 inversion.

Thus the proper extension for Theorem 5.3 is

Theorem 8.1. - Every conformal transtormation in $n$ dimensions can be expressed as the product of $r$ reflections and $s$ inversions, where

$$
r \leq n+1, \quad s \leq 2, \quad r+s \leq n+2 .
$$

In the language of pure inversive geometry, every conformal transforma. tion can be expressed as the product of $n+1$ or $n+2$ inversions. Thus was proved another way by RENÉ LAGRANGE [9, pp. 21.22, 112].

9. What happens when $n=2$ ? - The 2-dimensional case deserves special consideration for two reasons. First, since DupIs's Theorem is no longer available, we have to invoke the theory of functions of a complex variable in order to prove that every conformal transformation fof the whole inversive plane into itself) is a circle-preserving transformation. Secondly, elementary constructions are available for the invariant points of the two kinds of simi. larity, taken separately.

In fact, if a direct similarity is neither a translation, there must be at least one line that is transformed into a non-parallel line. Let $C$ be the common point of such a pair of lines, containing corresponding segments $A B, A^{\prime} B^{\prime}$. Then the invariant point $\mathrm{O}$ is the residual intersection of the two circles $A A^{\prime} C, B B^{\prime} C$. In other words, the triangles $O A B, O A^{\prime} B^{\prime}$ (possibly collapsing into triads of collinear points), being directly similar [CASEY 2, p. 180; FORDER 6, p. 16], are related by a spiral similarity: the product of a rotation about 0 and a dilatation from 0 . 
On the other hand, if the opposite similarity relating $A B$ and $A^{\prime} B^{\prime}$ is not a glide-reflection, let $A_{1}$ and $A_{2}$ divide $A A^{\prime}$ internally and externally in the ratio $A B: A^{\prime} B^{\prime}$, and let $B_{1}$ and $B_{2}$ divide $B B^{\prime}$ in the same manner. Then the lines $A_{1} B_{1}$ and $A_{2} B_{2}$ are perpendicular, and their common point $O$ is the desired invariant point. In other words, the triangles $O A B, O A^{\prime} B^{\prime}$, being oppositely similar [CASEY 2, p. 186; JoHNSON 7. p. 27], are related by a dilativereflection: the product of a reflection (in either $A_{1} B_{1}$ or $A_{2} B_{2}$ ) and a dilatation from $O$ [COXETER $4, \S 5.6]$.

These two entirely different kinds of similarity in the plane can both be derived from the one kind of spiral similarity in 3-dimensional space. Defining the 3-dimensional spiral similarity as in $\$ 4$, we see that it induces a 2-dimensional spiral similarity in the plane through 0 perpendicular to $l$. In the special case when the rotation about $l$ is a half-turn, there are infinitely many other invariant planes, namely all the planes through $l$. Any such plane is transformed according to a dilative-reflection.

The 2-dimensional analogue of Theorem 5.2 tells us that every confor, mal transformation of the whole inversive plane is either a spiral similaritya dilative-reflection, or the product of an inversion and an isometry. Of course, these are familiar as linear fractional transformations of a complex variable $z$ or of its conjugate $\bar{z}$. In particular, the product of an inversion and a reflection is elliptic, parabolic or hyperbolic according as the inverting circle and the reflecting line are intersecting, tangent, or non-intersecting; and the product of an inversion and a glide-reflection is loxodromic [FORD 1, p. 20].

\section{REFERENCES}

[1] E. Artin, Geometric Algebra, Interscience, New Jork, 1957.

[2] J. CAsEy, A sequel to the First Six Books of the Elements of Euclid, 6ith. ed., Longmans, London, 1892.

[3] H. S. M. Coxeter, Regular Polytopes, Methuen, London, 1948.

[4] H. S. M. Coxeter, Introduction to Geometry, Wiley, New York, 1961.

[5] L. R. Fond, Automorphic Eunctions; MoGraw Hill, Now York, 1929.

[6] H. G. Forder, Geometry, Hutchinson's University Library, London, 1950.

[7] R. Johnson, Modern Geometry, Houghton Mifflin. Boston, Mass., 1929.

[8] Lord Kelvin and P. G. TaIt, Treatise on Natural Philosophi, (I. 1)., Cambridge Uni. versity Press, 1888.

[9] R. Lagrange, Produits d'inversion et métrique conforme, Cahiers Scientifiques, 23 Gauthier-Villars, Paris, 1957. 